\title{
ON SECOND ORDER DIFFERENTIAL INEQUALITIES
}

\section{LLOYD JACKSON AND KEITH SCHRADER}

Introduction. Let $f\left(x, y, y^{\prime}\right)$ satisfy the following conditions:

(i) $f\left(x, y, y^{\prime}\right)$ is continuous on the slab

$$
S=\left\{\left(x, y, y^{\prime}\right)|a<x<b,| y|+| y^{\prime} \mid<+\infty\right\},
$$

(ii) for any $y_{1}$ and $y_{2}$ and any $a<x_{1}<x_{2}<b$ the boundary value problem

$$
\begin{aligned}
y^{\prime \prime} & =f\left(x, y, y^{\prime}\right), \\
y\left(x_{1}\right) & =y_{1}, \quad y\left(x_{2}\right)=y_{2}
\end{aligned}
$$

has a solution which extends throughout $(a, b)$ and any two solutions which agree at two distinct points are identical throughout $(a, b)$, and

(iii) solutions of initial value problems for (1) are unique.

In a paper in 1949 with these assumptions Peixoto [1] stated the following theorem.

Theorem 1. If $\phi \in C^{(2)}(a, b)$, then $\phi^{\prime \prime}(x) \geqq f\left(x, \phi(x), \phi^{\prime}(x)\right)$ on $(a, b)$ is a necessary and sufficient condition for $\phi$ to be a subfunction on $(a, b)$ with respect to solutions of (1).

The proof of the sufficiency of the differential inequality is based on $[1$, Lemma 1, p. 565] but the proof of the lemma does not appear to be correct. After some preliminary results in $\$ 1$, we give in $\$ 2$ a proof of Theorem 1 by a quite different method. Also in $\$ 2$ a modification of Theorem 1 is given in which the assumption of the solvability of the boundary value problem is replaced by a monotoneity condition.

1. Preliminary results. Let $F\left(x, y, y^{\prime}\right)$ be continuous for $c \leqq x \leqq d$, $|y|+\left|y^{\prime}\right|<+\infty$. Let $M>0, N>0$ be given and let

$$
Q=\operatorname{Max}\left\{\left|F\left(x, y, y^{\prime}\right)\right||c \leqq x \leqq d,| y|\leqq 2 M,| y^{\prime} \mid \leqq 2 N\right\} .
$$

Lemma 1. If $\left[x_{1}, x_{2}\right] \subset[c, d],\left|y_{1}\right| \leqq M,\left|y_{2}\right| \leqq M, \mid\left(y_{1}-y_{2}\right)$ $\left.\cdot\left(x_{1}-x_{2}\right)\right|^{-1} \leqq N$, and $\left|x_{1}-x_{2}\right| \leqq \operatorname{Min}\left[(8 M / Q)^{1 / 2}, 2 N / Q\right]$, then the boundary value problem

$$
\begin{aligned}
y^{\prime \prime} & =F\left(x, y, y^{\prime}\right), \\
y\left(x_{1}\right) & =y_{1}, \quad y\left(x_{2}\right)=y_{2}
\end{aligned}
$$

has a solution $y(x)$ of class $C^{(2)}\left[x_{1}, x_{2}\right]$.

Received by the editors March 24, 1966. 
The proof is a straightforward application of the SchauderTychonoff Fixed Point Theorem.

LEMma 2. If there are constants $h>0$ and $k>0$ such that

$$
\left|F\left(x, y, y^{\prime}\right)\right| \leqq h+k|y| 1 / 2
$$

for $c \leqq x \leqq d$ and $|y|+\left|y^{\prime}\right|<+\infty$, then the boundary value problem

$$
\begin{aligned}
y^{\prime \prime} & =F\left(x, y, y^{\prime}\right), \\
y(c) & =\gamma, \quad y(d)=\delta
\end{aligned}
$$

has a solution for all $\gamma, \delta$.

Proof. For a given $M>0$ and $N>0$ and $Q$ as defined above, we have

$$
Q \leqq h+k(2 M)^{1 / 2} .
$$

Hence, given $\gamma, \delta$ choose $M>0$ such that

$$
|\gamma| \leqq M,|\delta| \leqq M, \text { and }(d-c)^{2} \leqq 8 M /\left(h+k(2 M)^{1 / 2}\right) .
$$

Then with such an $M$ chosen and fixed choose $N>0$ such that

$$
|(\gamma-\delta) /(c-d)| \leqq N \text { and } d-c \leqq 2 N\left(h+k(2 M)^{1 / 2}\right) \text {. }
$$

It then follows from Lemma 1 that the boundary value problem has a solution.

Let $[c, d]$ be a compact subinterval of $(a, b)$ and let each of the functions $\phi$ and $\psi$ belong to $C^{(1)}[c, d]$ and $C^{(2)}(c, d)$. Assume that

$$
\psi^{\prime \prime} \leqq f\left(x, \psi, \psi^{\prime}\right) \text {, }
$$

and

$$
\begin{gathered}
\phi^{\prime \prime} \geqq f\left(x, \phi, \phi^{\prime}\right) \text { on }(c, d), \\
\psi(x) \geqq \phi(x) \text { on }[c, d] .
\end{gathered}
$$

For given functions $\phi$ and $\psi$ with the above properties let $N>0$ be such that

$$
N \geqq \operatorname{Max}\left[\operatorname{Max}\left|\phi^{\prime}(x)\right|, \operatorname{Max}\left|\psi^{\prime}(x)\right|\right] .
$$

Then define the function $F\left(x, y, y^{\prime}\right)$ as follows:

$$
\begin{aligned}
F\left(x, y, y^{\prime}\right) & =F_{1}(x, y, N) & & \text { for } y^{\prime}>N, \\
& =F_{1}\left(x, y, y^{\prime}\right) & & \text { for }\left|y^{\prime}\right| \leqq N, \\
& =F_{1}(x, y,-N) & & \text { for } y^{\prime}<-N,
\end{aligned}
$$

where 


$$
\begin{aligned}
F_{1}\left(x, y, y^{\prime}\right) & =f\left(x, \psi(x), y^{\prime}\right)+(y-\psi(x))^{1 / 2} \text { for } y>\psi(x), \\
& =f\left(x, y, y^{\prime}\right) \text { for } \phi(x) \leqq y \leqq \psi(x), \\
& =f\left(x, \phi(x), y^{\prime}\right)-(\phi(x)-y)^{1 / 2} \text { for } y<\phi(x) .
\end{aligned}
$$

The function $F\left(x, y, y^{\prime}\right)$ is continuous for $c \leqq x \leqq d,|y|+\left|y^{\prime}\right|<+\infty$ and $\left|F\left(x, y, y^{\prime}\right)\right| \leqq h+|y|^{1 / 2}$ where

$$
\begin{aligned}
h= & \operatorname{Max}\left\{\left|f\left(x, y, y^{\prime}\right)\right||c \leqq x \leqq d, \phi(x) \leqq y \leqq \psi(x),| y^{\prime} \mid \leqq N\right\} \\
& +\operatorname{Max}|\phi(x)|^{1 / 2}+\operatorname{Max}|\psi(x)|^{1 / 2} .
\end{aligned}
$$

Lemma 3. Let $\phi(x), \psi(x)$, and $F\left(x, y, y^{\prime}\right)$ be as above. Then for any $\gamma, \delta$ with $\phi(c) \leqq \gamma \leqq \psi(c)$ and $\phi(d) \leqq \delta \leqq \psi(d)$ the boundary value problem

$$
\begin{gathered}
y^{\prime \prime}=F\left(x, y, y^{\prime}\right), \\
y(c)=\gamma, \quad y(d)=\delta
\end{gathered}
$$

has a solution $y(x) \in C^{(2)}[c, d]$ and $\phi(x) \leqq y(x) \leqq \psi(x)$ on $[c, d]$.

Proof. It follows from Lemma 2 that the boundary value problem has a solution $y(x) \in C^{(2)}[c, d]$. Assume $y(x)>\psi(x)$ at some points of $(c, d)$, then $y(x)-\psi(x)$ has a maximum at some point $x_{0} \in(c, d)$. Then $y^{\prime}\left(x_{0}\right)=\psi^{\prime}\left(x_{0}\right)$ so that $\left|y^{\prime}\left(x_{0}\right)\right| \leqq N$, hence

$$
\begin{aligned}
& y^{\prime \prime}\left(x_{0}\right)-\psi^{\prime \prime}\left(x_{0}\right) \geqq f\left(x_{0}, \psi\left(x_{0}\right), \psi^{\prime}\left(x_{0}\right)\right)+\left(y\left(x_{0}\right)-\psi\left(x_{0}\right)\right)^{1 / 2} \\
& -f\left(x_{0}, \psi\left(x_{0}\right), \psi^{\prime}\left(x_{0}\right)\right)>0
\end{aligned}
$$

which contradicts having a maximum at $x_{0}$. Thus $y(x) \leqq \psi(x)$ and, similarly, $\phi(x) \leqq y(x)$ on $[c, d]$.

Lemma 4. Let $f\left(x, y, y^{\prime}\right)$ satisfy conditions (i) and (ii) as given in the Introduction. Let $\phi$ be continuous on the compact interval $[c, d] \subset(a, b)$ and assume that there is a solution $z(x)$ of (1) such that $z(c)=\phi(c)$, $z(d)=\phi(d)$, and $z(x)<\phi(x)$ on $(c, d)$. Then there is a solution $y(x)$ of (1) such that $y(c)>\phi(c), y(d)>\phi(d), y(x) \geqq \phi(x)$ on $(c, d)$ with equality holding at some point of $(c, d)$.

Proof. In [2] Beckenbach proves a number of results concerning familes of functions for which the boundary value problem is uniquely solvable. It follows from these results that if $\delta>0$ is sufficiently small the boundary value problem $y^{\prime \prime}=f\left(x, y, y^{\prime}\right), y(c)=\phi(c)+\delta, y(d)=\phi(d)$ will have a solution $u(x)$ with $u(x)<\phi(x)$ at some points in $(c, d)$. Furthermore, with such a fixed $\delta$ then for sufficiently large $M>\phi(d)$ the boundary value problem $y^{\prime \prime}=f\left(x, y, y^{\prime}\right), y(c)=\phi(c)+\delta, y(d)=M$ will have a solution strictly greater than $\phi$ on $[c, d]$. Let $M_{0}$ be the greatest lower bound of such $M$. Then the solution $y(x)$ of the 
boundary value problem with $y(c)=\phi(c)+\delta, y(d)=M_{0}$ satisfies the stated conditions.

2. Proof of Theorem 1. The proof of the necessity of a $C^{(2)}$ subfunction satisfying the differential inequality can be found in Theorem 1 of [1] or Theorem 6 of [3]. Consequently, we shall consider only the sufficiency.

Assume that $\phi \in C^{(2)}(a, b)$ and that $\phi^{\prime \prime} \geqq f\left(x, \phi, \phi^{\prime}\right)$ on $(a, b)$ but that $\phi$ is not a subfunction on $(a, b)$. It follows that there is a compact subinterval $[c, d] \subset(a, b)$ and a solution $z(x)$ of the differential equation such that $z(c)=\phi(c), z(d)=\phi(d)$, and $z(x)<\phi(x)$ for $c<x<d$. Then by Lemma 4 there is a solution $y(x)$ such that $y(c)>\phi(c)$, $y(d)>\phi(d), y(x) \geqq \phi(x)$ on $(c, d)$, and $y\left(x_{0}\right)=\phi\left(x_{0}\right)$ for some $c<x_{0}<d$. Let $N>0$ be chosen so that $\left|y^{\prime}(x)\right|<N$ and $\left|\phi^{\prime}(x)\right|<N$ on $[c, d]$, then with this $N, y(x)$, and $\phi(x)$, let $F\left(x, y, y^{\prime}\right)$ be the function employed in Lemma 3. It follows from Lemma 3 that with $\phi(c)<\gamma<y(c)$ and $\phi(d)<\delta<y(d)$ the boundary value problem

$$
\begin{gathered}
y^{\prime \prime}=F\left(x, y, y^{\prime}\right), \\
y(c)=\gamma, \quad y(d)=\delta
\end{gathered}
$$

has a solution $v(x) \in C^{(2)}[c, d]$ satisf ying

$$
\phi(x) \leqq r^{\prime}(x) \leqq y(x) \text { on }[c, d] .
$$

Then $\left|v^{\prime}\left(x_{0}\right)\right|=\left|y^{\prime}\left(x_{0}\right)\right|=\left|\phi^{\prime}\left(x_{0}\right)\right|<N$ and there is an open interval around $x_{0}$ in which $\left|v^{\prime}(x)\right|<N$. From the definition of $F$ it follows that

$$
F\left(x, v(x), v^{\prime}(x)\right)=f\left(x, v(x), v^{\prime}(x)\right)
$$

in such an interval and $v(x)$ is a solution of (1) in such an interval.

Let $(\alpha, \beta)$ be the maximal open subinterval of $[c, d]$ containing $x_{0}$ on which $\left|v^{\prime}(x)\right|<N$. As observed above $v(x)$ is a solution of (1) on $(\alpha, \beta)$ and, since $y\left(x_{0}\right)=v\left(x_{0}\right), y^{\prime}\left(x_{0}\right)=v^{\prime}\left(x_{0}\right)$ and the solution of the initial value problem for $(1)$ is unique, $v(x) \equiv y(x)$ on $(\alpha, \beta)$. Then $\left|v^{\prime}(\alpha)\right|=\left|y^{\prime}(\alpha)\right|<N$ and $\left|v^{\prime}(\beta)\right|=\left|y^{\prime}(\beta)\right|<N$ which forces us to conclude that $\alpha=c, \beta=d$, and $v(x) \equiv y(x)$ on $[c, d]$. This contradicts $v(c)<y(c)$ and $v(d)<y(d)$. We conclude that $\phi$ is a subfunction on $(a, b)$.

From the proof as given it is clear that it would have sufficed to assume that the solution of the initial value problem for (1) is unique on the right at every point or that it is unique on the left at every point.

Theorem 2. Assume $f\left(x, y, y^{\prime}\right)$ is continuous for $a \leqq x \leqq b,|y|+\left|y^{\prime}\right|$ 
$<+\infty, f\left(x, y, y^{\prime}\right)$ is nondeceasing as a function of $y$ for each fixed $x$ and $y^{\prime}$, and the initial value problem for $y^{\prime \prime}=f\left(x, y, y^{\prime}\right)$ has a unique solution. Then, if $\phi \in C^{(2)}(a, b), a$ necessary and sufficient condition that $\phi$ be a subfunction on $(a, b)$ is that $\phi^{\prime \prime} \geqq f\left(x, \phi, \phi^{\prime}\right)$ on $(a, b)$.

Proof. The proof of the necessity of the differential inequality is the same as in Theorem 1 and the references listed there still apply.

Assume that $\phi \in C^{(2)}(a, b)$ and $\phi^{\prime \prime} \geqq f\left(x, \phi, \phi^{\prime}\right)$ on $(a, b)$ but that $\phi$ is not a subfunction on $(a, b)$. Then again it follows that there is a compact interval $[c, d] \subset(a, b)$ and a solution $z(x)$ of the differential equation with $z(c)=\phi(c), z(d)=\phi(d)$, and $z(x)<\phi(x)$ on $(c, d)$.

Let $M=\operatorname{Max}\{\phi(x)-z(x) \mid c \leqq x \leqq d\}$, then $M>0$, and, if $\psi(x)$ $\equiv z(x)+M, \psi(c)>\phi(c), \psi(d)>\phi(d), \psi(x) \geqq \phi(x)$ on $(c, d)$ with equality at some point $c<x_{0}<d$, and $\psi^{\prime \prime} \leqq f\left(x, \psi, \psi^{\prime}\right)$ on $(c, d)$. Let $N>0$ be such that $\left|\psi^{\prime}(x)\right|<N$ and $\left|\phi^{\prime}(x)\right|<N$ on $[c, d]$. Let $F\left(x, y, y^{\prime}\right)$ be the function of Lemma 3 associated with $N, \phi$, and $\psi$. Then for $\phi(c)<\gamma<\psi(c), \phi(d)<\delta<\psi(d)$ the boundary value problem

$$
\begin{aligned}
y^{\prime \prime} & =F\left(x, y, y^{\prime}\right), \\
y(c) & =\gamma, \quad y(d)=\delta
\end{aligned}
$$

has a solution $y(x) \in C^{(2)}[c, d]$ with

$$
\phi(x) \leqq y(x) \leqq \psi(x) \text { on }[c, d] .
$$

Since $\left|\phi^{\prime}\left(x_{0}\right)\right|=\left|\psi^{\prime}\left(x_{0}\right)\right|=\left|y^{\prime}\left(x_{0}\right)\right|<N$, there is an open interval containing $x_{0}$ in which $\left|y^{\prime}(x)\right|<N$. Let $(\alpha, \beta)$ be the maximal such interval, then in $(\alpha, \beta) y(x)$ is a solution of $y^{\prime \prime}=f\left(x, y, y^{\prime}\right)$. If $a<\alpha$, then $\phi(\alpha)<y(\alpha)<\psi(\alpha)$. For, if for example $y(\alpha)=\phi(\alpha)$, then $\left|y^{\prime}(\alpha)\right|$ $=\left|\phi^{\prime}(\alpha)\right|<N$ and the interval $(\alpha, \beta)$ would not be maximal. Similarly, if $\beta<b$, then $\phi(\beta)<y(\beta)<\psi(\beta)$. Thus in any case $y(x)$ is a solution of $y^{\prime \prime}=f\left(x, y, y^{\prime}\right)$ on $[\alpha, \beta], y(\alpha)>\phi(\alpha), y(\beta)>\phi(\beta)$, and $y(x) \geqq \phi(x)$ on $[\alpha, \beta]$ with equality at $\alpha<x_{0}<\beta$. From this point the proof proceeds as in the proof of Theorem 1.

Again it suffices to have uniqueness of the initial value problem on the right or uniqueness on the left.

\section{REFERENCES}

1. M. M. Peixoto, Generalized convex functions and second order differential inequalities, Bull. Amer. Math. Soc. 55 (1949), 563-572.

2. E. F. Beckenbach, Generalized convex functions, Bull. Amer. Math. Soc. 43 (1937), 363-371.

3. Leonard Fountain and Lloyd Jackson, A generalized solution of the boundary value problem for $y^{\prime \prime}=f\left(x, y, y^{\prime}\right)$, Pacific J. Math. 12 (1962), 1251-1272.

UNIVERSITY OF NEBRASKA 